\title{
Investigation Of The Bragg-Snell Law In Photonic Crystals
}

\author{
Gina Mayonado*, Shabbir M. Mian*, Valentina Robbiano† and Franco Cacialli† \\ *Department of Physics, McDaniel College, 2 College Hill, Westminster, MD 21157 \\ †Department of Physics and Astronomy, and London Centre for Nanotechnology, University College London, \\ Gower Street, London, WC1E 6BT, United Kingdom
}

\begin{abstract}
We present an optical experiment on photonic crystals suitable for an advanced physics laboratory course or a senior capstone project. Photonic crystals are periodically ordered composite systems made of materials that have different dielectric constants, and can be arranged in one, two, or three dimensions. They are characterized by a bandgap that depends on the size, arrangement and dielectric constant of the microstructures that make up the crystal. In addition, the bandgap spectrally shifts with the angle of incident light. These observations are captured by the Bragg-Snell law. In this paper, we describe a vertical deposition method for growing photonic crystals from a water suspension of polystyrene microspheres, as well as a simple transmission experiment that students can perform using a USBspectrometer to explore the Bragg-Snell law.
\end{abstract}

Keywords: Spectroscopy, Photonic Crystals, Bandgap, Bragg-Snell Law PACS: 78.67.Pt, 42.70.Qs, 01.50.Pa

\section{INTRODUCTION}

In this paper, we present a simple spectroscopic experiment on photonic crystals (PhCs) suitable for an advanced physics laboratory course or senior capstone project. PhCs are periodically ordered composite media made of materials having different dielectric constants, and can be arranged in one, two or three dimensions. ${ }^{1}$ The periodicity is comparable to the wavelength of visible light, which allows for an incident beam to be strongly diffracted. This behavior is governed by the Bragg-Snell law that states that the wavelength of the diffracted light depends on the angle of incidence, the periodicity, and effective refractive index of $\mathrm{PhC}$. $^{2}$ It is, therefore, possible to construct a PhC that prevents light from propagating in certain directions with specified frequencies. These forbidden spectral regions are called photonic bandgaps (PBGs). In that sense, the behavior of photons in photonic crystals is similar to the behavior of electrons in semiconductors.

PhCs have been extensively studied since their discovery in $1987,,^{3-4}$ and engineered for a variety of applications such as photovoltaics and optical microchips, ${ }^{5}$ metamaterials, ${ }^{6}$ and holey fibers, ${ }^{7}$ to name a few. In addition to being artificially created, $\mathrm{PhCs}$ also occur in nature such as neck feathers in domestic pigeons, ${ }^{8}$ certain beetles, ${ }^{9}$ and panther chameleons. ${ }^{10}$
Studying the nature of PhCs with relation to the Bragg-Snell law provides a good understanding of the wave nature of light-matter interaction, and is something that is easily accessible to undergraduate students. For example, creating PhCs with the same material and crystalline structure but different periodicity will produce the same effective refractive index for the incident light, a fact that students can easily verify experimentally. Furthermore, it is striking to see the tuning of the bandgap with the angle of incidence in accordance with the Bragg-Snell law in real-time using commercial USB CCD spectrometers.

The paper is organized as follows. We present an outline for the derivation of the Bragg-Snell law, provide an overview on how to grow PhCs with polystyrene microspheres (artificial opals), describe our experimental setup, review our results on PhCs of different sizes, and finally offer suggestions for other explorations of the Bragg-Snell law.

\section{THEORY}

$\mathrm{PhCs}$ prevent light over a small frequency range from passing through the crystal in specific directions by destructive interference. The light is, thus, back diffracted and it will appear as a reflection peak whose frequency corresponds to the PBG. The reflection 
maximum or, equivalently, transmission minimum, can be shifted by varying the angle of incidence, the lattice spacing or material composition. These observations are described by the Bragg-Snell law,

$$
m \lambda=2 D \sqrt{n_{e f f^{2}}-\sin ^{2} \theta},
$$

where, $m$ is the order of diffraction, $\lambda$ is the free space wavelength of light, $D$ is the interplanar spacing, $n_{\text {eff }}$ is the effective refractive index, and $\theta$ is the angle of incidence of light with respect to the normal. Fig. 1 shows a PhC of spherical particles with the pathlength light rays take during the diffracting process. The

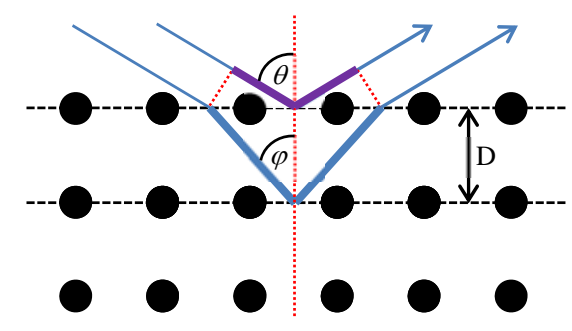

FIGURE 1. This figure shows how incident light undergoes diffraction when interacting with a photonic crystal. The pathlength difference between the two rays is $m \lambda$.

pathlength difference between the two rays must be an integer multiple of wavelengths for strong diffraction.

The Bragg-Snell law can be derived from an application of Bragg's law of diffraction,

$$
m \lambda=2 D \sin \theta,
$$

and Snell's law of refraction,

$$
n_{1} \sin \theta=n_{2} \sin \varphi \text {, }
$$

where $n_{2}$ is the refractive index of the microstructures, $n_{1}$ is the refractive index of the interstitial medium, and $\varphi$ is the angle of the refracted ray in the crystal. The effective refractive index is related to the sum of the refractive indices of the two media, weighted by their volume fraction, through the Lorentz-Lorenz relation ${ }^{11}$,

$$
\frac{n_{e f f}^{2}-1}{n_{e f f}^{2}+2}=f \frac{n_{p s}^{2}-1}{n_{p s}^{2}+2}+(1-f) \frac{n_{a i r}^{2}-1}{n_{a i r}^{2}+2} .
$$

Artificial opals have a face-centered cubic structure and the volume fraction, $f$, for spheres is $0.74 .^{12}$ In our case, we used polystyrene microspheres $\left(n_{2}=n_{p s}=\right.$ $1.59)$ in air $\left(n_{1}=n_{\text {air }}=1.00\right)$. Based on Equation (4), the effective refractive index is 1.41 for artificial opals regardless of the size of the microspheres.

\section{OPAL GROWTH}

We prepared the artificial opals from commercial monodisperse polystyrene microspheres in a water suspension (Thermo Scientific) with the following diameters: 200, 220, and $370 \mathrm{~nm}$. The microspheres occupied $10 \%$ of the volume, and the standard deviation of the particle diameters was less than 5\%. In order to create opals of the desired thickness, we diluted the suspensions to a concentration of $0.2 \%$ volume/volume by adding $4.9 \mathrm{ml}$ of deionized water to $100 \mu \mathrm{l}$ of suspension for a total of $5 \mathrm{ml}$. In our experience, an opal thickness of 2-3 microns seems to offer the best compromise between reflection and transmission, and allows the study of optical properties of polymers infiltrated into the opals. ${ }^{13}$

We grew the opals at $28{ }^{\circ} \mathrm{C}$ inside an incubator (Gentec) using the vertical deposition method. ${ }^{14}$ The substrates were commercial soda lime slides, and were cleaned with the RCA-1 procedure to give it a more hydrophilic surface. ${ }^{15}$ We attached the slides to a dipcoater consisting of a motorized translation stage (Thorlabs), and immersed them in the microsphere suspension as shown in Figure 2 (a). The stage moved up at a controlled rate of $7 \times 10^{-5} \mathrm{~mm} / \mathrm{s}$ and the growth process took about three days.
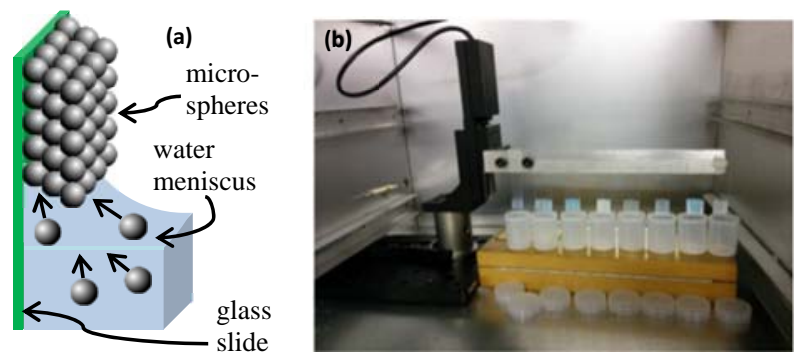

FIGURE 2. (a) In the vertical deposition method, the film forms at the water meniscus and glass substrate interface. The [111] direction is perpendicular to the substrate. (b) The dip-coater assembly can handle multiple slides and opal growth takes about 3 days.

Fig. 2(a) shows the basic growth mechanism. The opal film is created at the interface between the water meniscus and the glass substrate by the Van der Waals force during water evaporation. The polystyrene microspheres self-assemble into a face-centered cubic lattice with the crystallographic [111] direction oriented perpendicular to the substrate. In order to avoid the presence of stacking faults, we placed the entire set-up on an optical table to minimize vibration. Figure 2(b) shows the dip-coater inside the incubator with multiple slides securely attached to an extension. This allows us to simultaneously create opals of different sized microspheres. 


\section{TRANSMISSION EXPERIMENT}

The experimental setup shown in Fig. 3 consists of a tungsten-halogen white light source (360-2000 nm, Ocean Optics LS-1) that was slightly apertured before collimating. This is an important step that limits the range of incident angles of the rays comprising the incident beam, and simplifies the analysis of the resulting spectra.

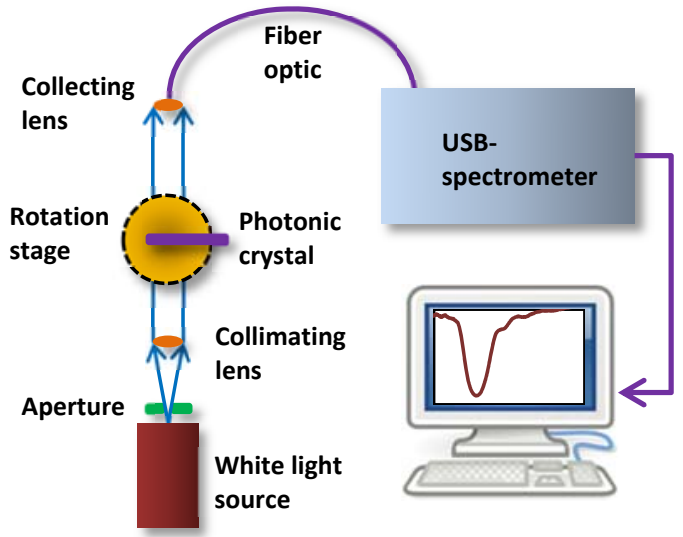

FIGURE 3. Experimental setup for measuring transmission spectra of the PhC.
We placed the opal on a rotation stage (Thorlabs, RP01, $2^{\circ}$ markings) and ensured that the incident light hit the crystal on the rotation axis. We collected the scattered light using a fiber-optic cable connected to a computer-controlled USB spectrometer (Ocean Optics, MayaPRO 2000). We adjusted the angle of incidence in $4^{\circ}$ increments from $0^{\circ}$ up to $44^{\circ}$, and measured the wavelength for minimum transmission for each angle.

\section{DISCUSSION}

The bandgap in artificial opals only exists in the [111] direction, and is called a pseudo-bandgap ( $p$ PBG). At the $p$-PBG, light is coherently diffracted by the (111) planes giving rise to a transmission minimum. ${ }^{16}$ Fig. 4 shows the transmission spectra for the opals formed by (a) $200 \mathrm{~nm}$, (b) $220 \mathrm{~nm}$, and (c) $370 \mathrm{~nm}$ microspheres. Notice that the photonic bandgap shifts to smaller wavelengths (higher energy) with increasing incidence angle in accordance with the Bragg-Snell law. The transmission spectra have broad spectral features and the shifts are also quite large. As such, they can be easily measured using low-cost USBspectrometers. For the maximum angle of $44^{\circ}$ we used in our experiment, the shift was about $50 \mathrm{~nm}$ for 200 $\mathrm{nm}$ opal, $56 \mathrm{~nm}$ for $220 \mathrm{~nm}$ opal, and $108 \mathrm{~nm}$ for 370 nm opal.
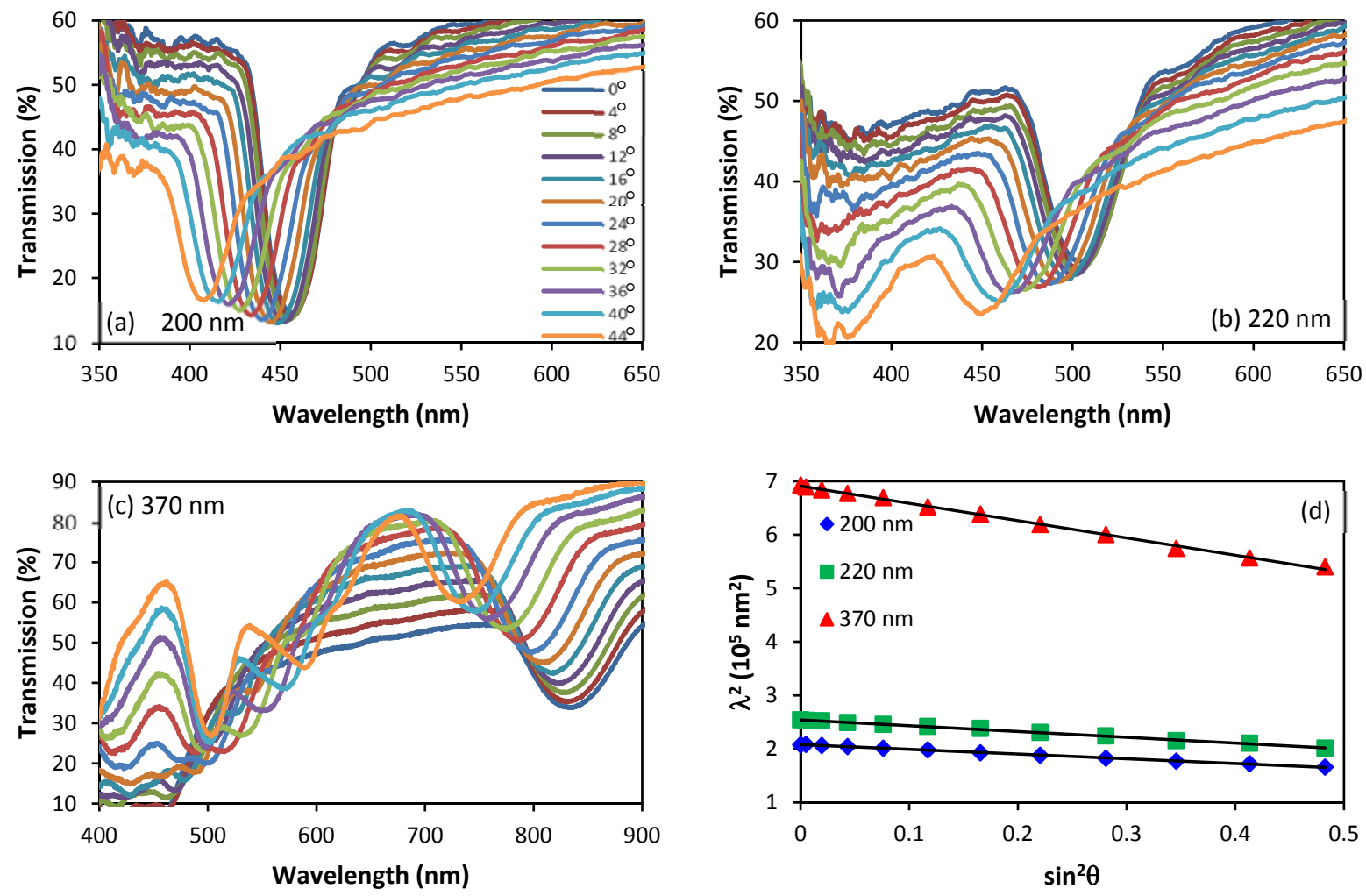

FIGURE 4. This figure shows transmission spectra of the opals as a function of incidence angle for (a) $200 \mathrm{~nm}$, (b) $220 \mathrm{~nm}$ and (c) $370 \mathrm{~nm}$. The Bragg-Snell law analysis of the spectra appears in (d) where the lines are linear regressions. 
Fig. 4 (d) shows a linearized form of Bragg-Snell law applied to the minimum transmission wavelength and incident angle. The solid lines through the data are linear regressions from which we derive an effective refractive index of 1.40 for each opal in good agreement with the Lorentz-Lorenz relation.

Notice for the $370 \mathrm{~nm}$ opal (Fig. $4 \mathrm{c}$ ), the $p$-PBG lies in the near-IR and there are additional structures that appear in the transmission spectra at lower wavelength. These are caused by diffraction from planes in the opal other than (111). They are called van Hove singularities and their dispersion is different to that of $p$-PBG. While the $p$-PBG moves to lower wavelength, the van Hove singularity peaks show opposite behavior. ${ }^{17}$ Observation of van Hove singularities correlate with high quality PhCs.

\section{CONCLUSION AND SUGGESTIONS}

We presented a simple transmission experiment on artificial opals suitable for undergraduate students. Since creating the opals can take up to three days, it can be done beforehand by the instructor if time is a factor. In addition to finding the effective refractive index from the Bragg-Snell law, we suggest several other interesting investigations. (1) Students can look at reflection spectra instead of transmission spectra as a function of incident angle. This requires a slight modification in the geometry presented in Fig. 3. (2) They can also measure the opal thickness and homogeneity from interference fringes in the normal reflection spectrum. The thickness can be correlated to SEM images, if available. (3) Another interesting study is the effects of polarization on transmission and/or reflection spectra. For example, the minimum in the transmission spectrum increases for p-polarized light as the incident angle approaches Brewster's angle. (4) Students can study the effects of changing the microsphere material on the $p$-PBG. (5) Finally, students can correlate diffraction from van Hove singularities to $p$-PBG for larger microsphere opals. This last suggestion requires students to learn about density of states, which is an interesting and crucial topic for understanding photonic crystals.

\section{ACKNOWLEDGMENTS}

S. M. Mian would like to thank the Faculty Development Committee of McDaniel College for partial financial support of this project. V. Robbiano and F. Cacialli would like to thank the EU HORIZON
2020 Research and Innovation Programme under Grant Agreement N. 643238 (SYNCHRONICS) and the EC Seventh Framework Programme PITN-GA-2012317488-CONTEST. F. Cacialli is a Royal Society Wolfson Research Merit Award holder.

\section{REFERENCES}

1. J. D. Joannopulos, R. D. Meade, and J. N. Win, Photonic Crystals: Molding the Flow of the Light, 2nd ed. (Princeton University Press, Princeton, 2008), pp 1-5.

2. Geoffrey A. Ozin, André C. Arsenault, Ludovico Cademartir, Nanochemistry: A Chemical Approach to Nanomaterials, 2nd ed. (Royal Society Chemistry, Cambridge, 2009), pp 462-464.

3. E. Yablonovitch, Physical Review Letters 58, pp. 20592062 (1987).

4. S. John, Physical Review Letters 58, pp. 2486-2489 (1987).

5. S. John, Nature Materials, 11, pp. 997 (2012)

6. S. Foteinopoulou, Physica B 407, pp. 4056-4061 (2012).

7. E. Yablonovitch, Scientific American, 12, pp. 47-55 (2001).

8. H. Yin, L. Shi, J. Sha, Y. Li, Y. Qin, B. Dong, S. Meyer, X. Liu, L. Zhao, and J. Zi, Physical Review E 74, 051916 (2006).

9. J. Vigneron and P. Simonis, Physica B 407, pp. 40324036 (2012).

10. J. Teyssier, S. V. Saenko, D. van der Marel and M. C. Milinkovitch, Nature Communications 6, 6368 (2015).

11. Charles Kittel, Introduction to Solid State Physics, $8^{\text {th }}$ ed. (Wiley, Hoboken, 2005), p. 464.

12. D. Comoretto, R. Grassi, F. Marabelli, L. C. Andreani, Growth and optical studies of opal films as threedimensional photonic crystals. Materials Science and Engineering C, 23, pp. 61-65 (2003).

13. F. Di Stasio, L. Berti, S. O. McDonnell, V. Robbiano, H. L. Anderson, D. Comoretto, and F. Cacialli, Applied Physics Letters - Materials 1, 042116 (2013).

14. P. Jiang, J. F. Bertone, K. S. Hwang, and V. L. Colvin, Chemistry of Materials, 11, 2132-2140 (1999).

15. B. Fuhrmann, H. S. Leipner, H.-R. Höche, L. Schubert, P. Werner, and U. Gösele, Nano Letters 5, pp. 2524-2527 (2005).

16. C Lopez, Journal of Optics A: Pure and Applied Optics 8 R1-R14 (2006).

17. E. Pavarini, L. C. Andreani, C. Soci, M. Galli, F. Marabelli, and D. Comoretto, Physical Review B 72, 045102 (2005). 